\title{
PERSON RE-IDENTIFICATION USING DEEP METRIC LEARNING
}

\author{
Ms. Shruti Jalapur \\ Department of CSE \\ SECAB Institute Vijayapura, Karnataka, India
}

\author{
Ms.Bibi Ayesha Hundekar \\ Department of CSE \\ SECAB Institute Vijayapura,Karnataka,India
}

\begin{abstract}
Today everyone faces a multitude and variety of threats ranging from robbery, kidnapping and terrorism to murder. To avoid these threats, authorities need to collect real-time information about what's going on in and around the city. New technologies are therefore being developed to make cities safer and more risk-free. Here we have built a reliable system that recognizes the person from every angle from a recorded image. We can get the input into the systems through CCTV cameras installed in public places where these types of life threatening events take place. It is easy to install these cameras in public places, and it is easier to monitor and store the data. The developed system uses deep metric learning and the machine learning platform, Tensor Flow and Keras. It's a type of machine learning where the system iteratively performs calculations to know the patterns. The system processes recorded images and compares them with existing data records in order to identify the person. The comparison is made based on certain selected features. The results are more accurate $(\mathbf{9 8 . 1 8 \%})$ ) compared to existing systems.
\end{abstract}

Keywords-Person Re-identification, Deep metric Learning, CNN

\section{INTRODUCTION}

An Introduction to Machine Learning: Machine learning is a branch of Artificial Intelligence (AS). For this reason, machine learning focuses on developing computer programs that can access and use data to learn on their own. Machine learning has been a buzzword for several years Arthur Samuel, an American IB Mer and pioneer in the field of computer games and artificial intelligence, coined the phrase machine learning in 1959. In 1997, Tom M. Mitchell published a widely referenced, more formal explanation of the machine learning algorithms: "In regard to a task $\mathrm{T}$, a computer programme should learn from experience E. and quantify some performance.

Machine learning is a type of deep metric learning. Deep metric learning (DML) is a distance-metric learning paradigm that combines deep learning and metric learning.. this requires that computer-systems perform iteratively calculate to identify patterns themselves. This means after a deep learning computer recognize that an image it is evaluated has the shape of a rectangle, oval or pattern by going through $\mathrm{n}$ cycles, and therefore our project will use the same logic as that Person re-identification applied via deep metric learning, in which it goes through $\mathrm{n}$ cycles to identify a person by assessing the similarities in relation to the data provided and finally presenting the positive results.

To make this possible, we use Torchried, the pytorch-based library for deep metric learning, which is specifically considered for the identification of a person.

\section{PROPOSED AlgorithM}

Implementation of this project is divided into three modules 1. Dataset preparation:

- Three classes are made namely a,b and c

- The class ' $a$ ' contains images of $x$ person, class ' $b$ ' contains images of y person and class c contains images of ' $z$ ' person

- All the classes are trained with their respective images around a data of 1400 images are trained

- And a own dataset is created

2. Training the images:

- After collecting the database the next procedural step is to tain thedata

- This is an major act to gather the data by collecting the images and training atleast for 1 hour for a good accuracy

- Duplicating the images hence for the better learning of the machine

3. Analyzing the data:

- In this step we analyse the data and try to match it out with the present database

- And once the needed picture matches with the given database the final upshot can be presented

- And hence the last step is the final decision of identifying a person

We used the transfer-learning technique, Mobilenet with image net weights as the base model with frozen upper layers, we added new layers to the base model and refined them on our user-defined data set,

1. MobileNet is an image classification model, it is a neural network with dense convolutional layers and pooling / subsampling layers. Compared to other 
similar image classification models / algorithms like vgg116, densenet, Xception, .. etcyolo gives better accuracy.

2. In combination with the mobileet image classification model, we added a few new levels and retrained it with new data adapted to our images. The accuracy when training the model was $99.6 \%$

3. The new model can be used to process the image pixels as tensors flowing out of the top network layer and making the predictions

4. The model is then saved as model.h5 and model.json and can be loaded with load weights and used for predictions.

5. The saved model takes the paths of the images and converts them into corresponding pixel values and makes them available to the saved model with the function [model.predict].

6. We used opencv to read and display the input images from the system path. The output is specified as ["TRUE" / "FALSE"] values.

Transfer Learning (TL) is a machine learning (ML) research problem that well defined on the answer one issuse and then apply that knowledge to a different but related problem. For example, learn to identify cars when they are used to identify knowledge trucks. Although the two areas of formal relationships are limited, this area of research has some references to the long history of the psychological literature on learning transfer. From a practical point of view, reinforcement learning resources are the recycling or transfer of information about learning tasks that are new to the task.

\section{EXPERIMENT AND RESULT}

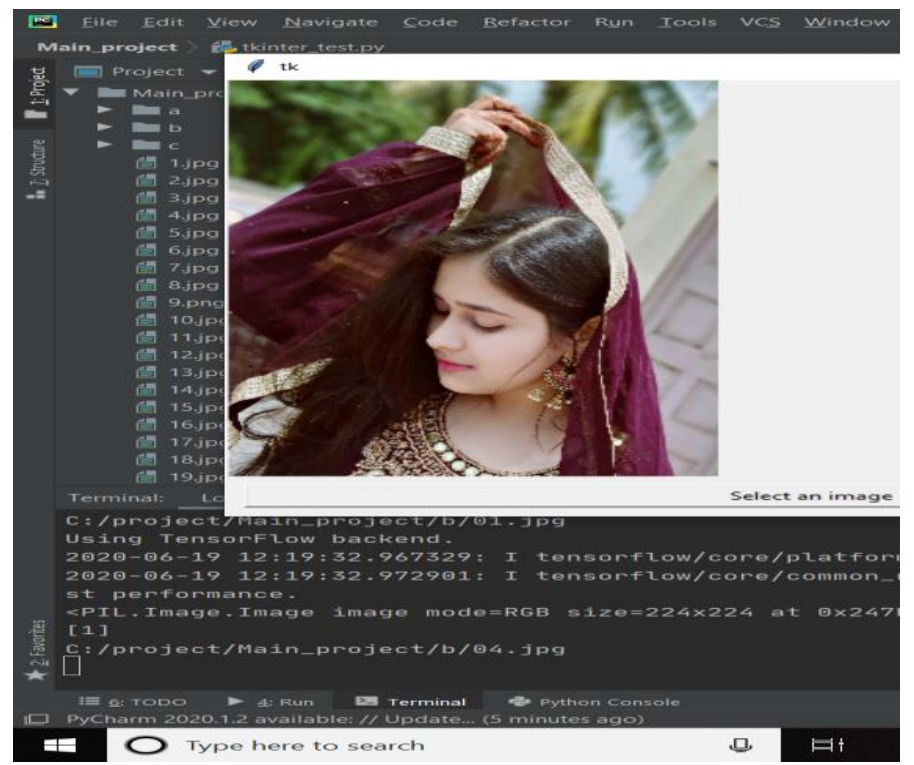

figure 1: Beforedetection
This is a sample image that we nourish on the algorithm and expect our algorithm to determine and recognize the image and predict the output of this image, that is, identify whether it is the same person or not, in accordance with the appropriate class.

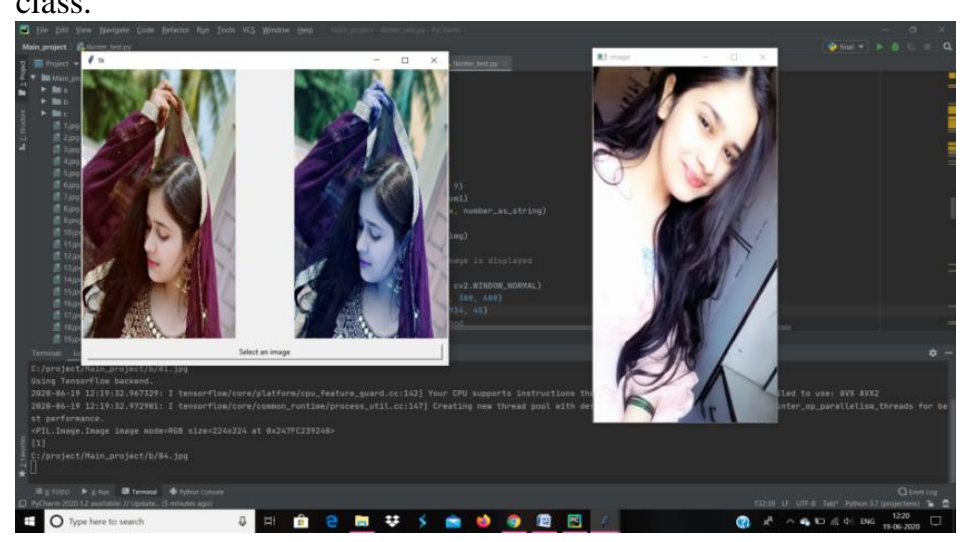

\section{figure 2 After detection}

Depending on your needs, our algorithm recognizes the image based on its classes and nominates the image based on its tag and determines the dimensions of the image.

Input kind :We also describe and analyze the File trail or pathway for a image, a numpy array, rather a File track or Stream for a image as an Input Image.

Output kind : We also describe for the "Detect Image FromImage" operation have to deliver the Image of the formation with file or a numpy array.

In this segment a complete experimental similarity of the different face recognition algorithms has been introduced. The numerous performance metrics for examining the results are accuracy, precision, detection rate, and false detection rate. Accuracy indicates the proportion of true results, both true and negative. The accuracy is recorded efficiently.

The experiment shows excellent results for the proposed algorithm. The low false detection rate indicates that the algorithm can distinguish between the actual skin color and background color with the appearance of the skin color. The recognition rate is $98.18 \%$. 
International Journal of Engineering Applied Sciences and Technology, 2021

Vol. 6, Issue 4, ISSN No. 2455-2143, Pages 361-364

Published Online August 2021 in IJEAST (http://www.ijeast.com)

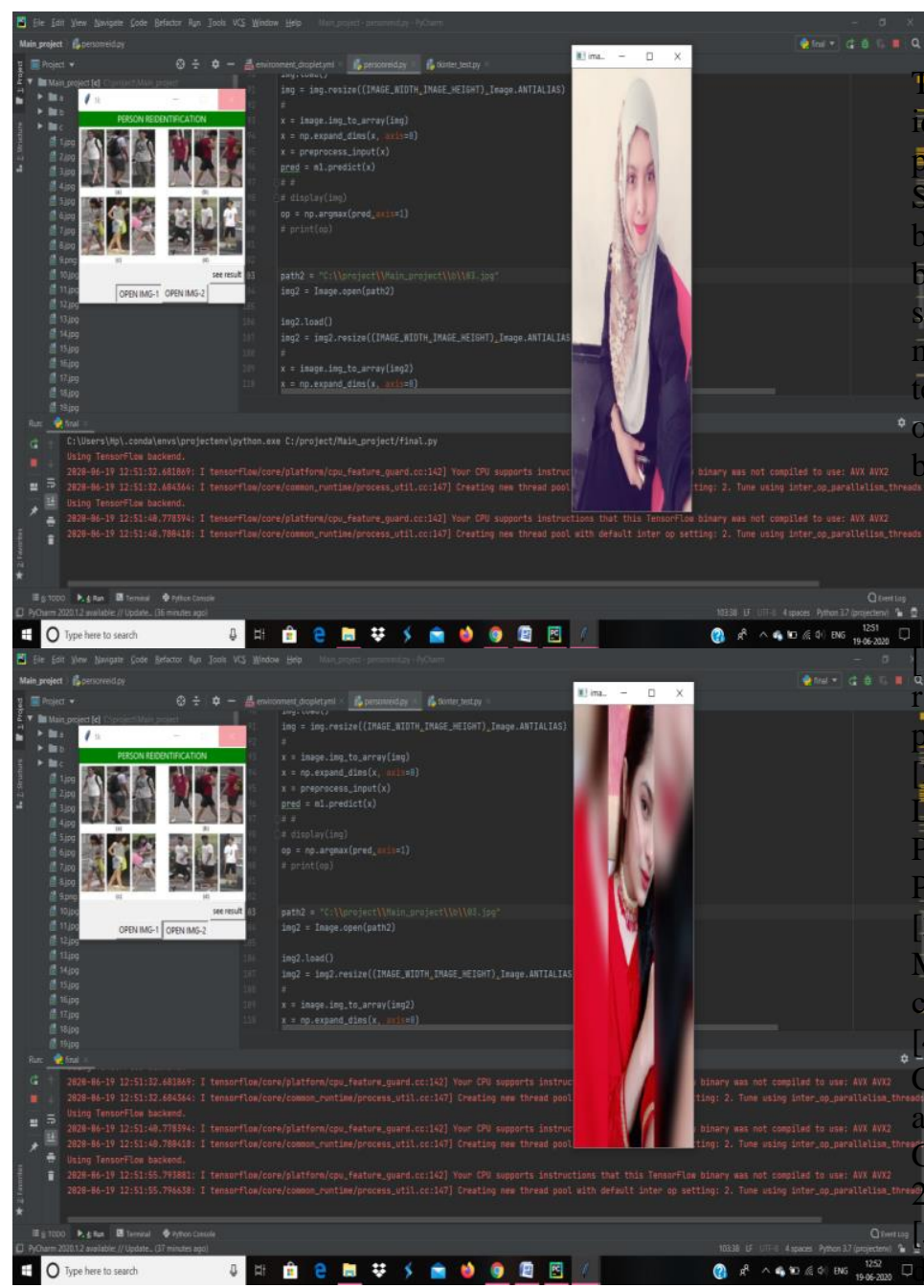

\section{CONCLUSION}

This paper illustrates the process related to person redentification. It uses deep metric learning for this same urpose. The Decorrelated Centralized Loss with Normalizecale algorithm provides an accuracy of $98.18 \%$. This offers etter efficiency compared to existing methods. This system is etter suited for tracking down criminals in criminal matters uch as robbery, terrorist attacks, intruder detection and much hore. Its efficiency can be further increased by using ensorflow and keras as a library and module internet with its wn organized data sets. This can be a new approach that can e implemented in the future.

\section{REFERENCE}

] R. Layne, TM Hospedales and S. Gong, "Attributes-based eidentification", in Person Re-Identification Springer, 2014, p. 93-117.

2] E. Ahmed, M. Jones and TK Marks, "An Improved Deep earning Architecture for Person Re-Identification", in roceedings of the Conference on Computer Vision and attern Recognition, 2015, pp. 3908-3916.

3] Liang Zheng, Hengheng Zhang, Shaoyan Sun, ManmohanChandraker, and QiTian, "Personre-identi fi ationinthewild," in CVPR, 2017.

4] M. Farenzena, L. Bazzani, A. Perina, V. Murino and M. rristani, "Person re-identi fi cation by symmetry-driven ccumulation of local features", 2010 IEEE Computer Society Fonference on Computer Vision and Pattern Recognition, 010, pp. 2360-2367.

5] SakrapeePaisitkriangkrai, ChunhuaShen and Anton van en Hengel, "Learningtorankinpersonre-identi fi

Models are evaluated using a test data set. The trained test set consists of the three classes "a, b \& c", which include images of 3 different peoples with different backgrounds, objects and different image views. As can be seen, the class distribution in these test data was the same as in the training data. We get this security to measure approx. Generalized performance of the model. The main use of our Iq ${ }^{\circledR} \mathrm{xq}$ projection (image $\mathrm{q}$ to embedding q) is to look up visually similar images.

After comparing the images, the algorithm returns TRUE or FALSE. If the compared images are TRUE or equal (checking the path given to the images in the algorithm), TRUE is returned, if not, the result is returned as FALSE.

The results of various experiments are discussed in this results and discussion section. The outcome describe the advance algorithms presents stronger contract for the other algorithms since we created our own datasets in this project. The results of the proposed algorithm are also used for applications such as pose and expression. cationwithmetric ensembles", in Proceedings of the IEEE Conference on Computer Vision and Pattern Recognition, 2015, pp. 1846-1855.

[6] S. Liao, Y. Hu, X. Zhu and SZ Li, "Person reidenti fi cation by local maximum instance Representative and metric learning", in CVPR, 2015.

[7] Bedagkar-Gala, A. and SK Shah, An overview of approaches and trends in person re-identification. Image and image processing, 2014. 32 (4): p. 270-286.

[8] AlexKrizhevsky, IlyaSutskever and Geoffrey E. Hinton, "Imagenet classi fi cation with deep convolutional neural network", in Advances in neural information processing systems, 2012, pp. 1097-1105.

[9] YC Chen, WS Zheng, JH Lai and P. Yuen, "An Asymmetrical Distance Model for the Depiction of CrossSighting Features in Person Re-Identification", IEEE Transactions on Circuits and Systems for Video Technology, vol. PP, No. 99, pp. 1-1, 2016.

[10] R. Kawai, Y. Makihara, C. Hua, H. Iwama and Y. Yagi, "Person reidenti fi cation using view-dependent score-level 
fusion of gait and color features", in IKSR, 2012, p. 2694 2697.

[11] D. Tao, Y. Guo, B. Yu, J. Pang and Z. Yu, "Deep MultiView Feature Learning for Person Re-Identification", in IEEE Transactions on Circuits and Systems for Video Technology, vol. 28, No. 10, p. 26572666, 2018.

[12] H. Han, AK Jain, X. Chen, F. Wang and S. Shan, "Heterogeneous Face Attribute Estimate: A deep multi-task learning approach" IEEE Trans. Muster Anal. Do. Intellect., Vol. 40, no. 11, pp. 2597-2609, Nov. 2018. 